Aus: Bibliotheksdienst,

Band 39, Heft 1, Seiten 76-77,

THEMEN ISSN (Online) 2194-9646

ISSN (Print) 0006-1972,

DOI: https://doi.org/10.1515/bd.2005.39.1.76

Bibliotheken

\title{
Zum Beitrag „Über die Zukunft der namenlos gemachten Bibliothek"'1
}

\section{Andreas Degkwitz}

Zu dem von Ulrich Naumann mit o. g. Beitrag angesprochenen Thema ,Informationsmanagement' wird es noch mehr Diskussion geben, was im Hinblick auf die Weiterentwicklung von Bibliotheken und Informationseinrichtungen notwendig und wichtig ist. Aus Sicht des in diesem Kontext genannten Informations-, Kommunikations- und Medienzentrums (IKMZ) der BTU Cottbus ist dazu festzustellen:

Ob Fortbestand, Überlebens- und Zukunftsfähigkeit von Bibliotheken abhängig sind von Namen oder Bezeichnungen? Die Ziele der bibliothekarischen Weiterentwicklung, die der Beitrag vermittelt, stimmen jedenfalls mit denen des IKMZ überein. Auch in Cottbus gilt weiterhin der Grundauftrag und das Grundverständnis bibliothekarischer Aufgaben, das „seit 5000 Jahren gilt: sammeln, ordnen und verfügbar machen". Zugleich ist die Bibliothek der größte Teilbereich des IKMZ und vom Gebäude her unübersehbar präsent ${ }^{2}$. Anders ist allerdings die organisatorische Einbindung der Bibliothek in die Informationsinfrastruktur der Universität: Welche Ziele verbinden sich mit dem IKMZ?

Im Grunde geht es darum, die Dienste- und Servicestruktur der zentralen Einrichtungen zu vereinheitlichen, um Zugang und Zugriff auf die Informations-, Literatur- und Medienangebote der Universität zu vereinfachen und um die Arbeitsumgebung für Leser und Nutzer zu optimieren. Die im IKMZ zusammengefassten Einrichtungen Bibliothek, Multimediazentrum, Rechenzentrum und Verwaltungs-Datenverarbeitung bringen dafür ihre jeweiligen Kernkompetenzen ein. Um die damit verbundenen Aktivitäten besser zu koordinieren, wurden die vier Bereiche unter eine gemeinsame Leitung zusammengeführt. Von der Universität werden darin bessere Erfolgchancen für die Weiterentwicklung inrer Informationsinfrastruktur gesehen, als wenn diese vier Bereiche als eigenständige Einrichtungen kooperieren. Zu dem von Ulrich Naumann prognostiziertem „Kompetenzwirrwarr" hat diese Entwicklung nicht geführt; auch sind die einzelnen Teilbereiche organisatorisch abgesichert und weiterhin wahrnehmbar.

1 in: BIBLIOTHEKSDIENST 38 (2004), S. 1399-1416

2 vgl. http://www.ikmz.tu-cottbus.de mit weiterführenden Informationen und Bildgalerie 
Der mit dem IKMZ verfolgte Ansatz ist nutzerorientiert und in Anbetracht des neuen IKMZ-Gebäudes auch bibliotheksorientiert. Die Bibliothek ist mit den anderen Einrichtungen in die gemeinsame Weiterentwicklung der universitären Informationsinfrastruktur eingebunden, was ihre Rolle stärkt und die Transformation zur Hybrid-Bibliothek unterstützt, ohne dass deshalb die Versorgung mit gedruckten Medien in den Hintergrund tritt. Aufgrund des Profils der BTU Cottbus liegt allerdings ein Schwerpunkt auf digitalen Formen der Literatur- und Informationsversorgung.

Selbstverständlich versteht sich das IKMZ als ein (jedoch nicht als das einzige) Modell, das die sicher notwendige Kooperation zwischen Bibliothek, Medien- und Rechenzentrum und Verwaltungsdatenverarbeitung sicherstellt; zugleich hat der in Cottbus verfolgte Weg auch experimentellen Charakter. Eine „allkompetente Einheitseinrichtung für das Informationsmanagement" ist das IKMZ nicht. Im Mittelpunkt aller Aktivitäten stehen nutzerorientierte Dienste und deren gemeinsame Verbesserung: Viele Wege führen nach Rom - an der BTU Cottbus heißt er IKMZ! 